\title{
HEALTH BEHAVIOR AND URINARY TRACT INFECTION IN COLLEGE-AGED WOMEN*
}

\author{
BETSY FoXMAN ${ }^{1}$ and JEN-Wer CHI ${ }^{2}$ \\ Departments of ${ }^{1}$ Epidemiology and ${ }^{2}$ Biostatistics, University of Michigan School of Public Health, \\ Ann Arbor, MI 48109, U.S.A.
}

(Received in revised form 5 September 1989)

\begin{abstract}
We conducted a case-control study to examine the associations between various behavioral risk factors and urinary tract infection among college-aged women. Cases were collected from a University Health Service, and were compared to Health Service controls and to a population-based control group. Sexual intercourse, diaphragm use, and urinating after sexual intercourse were each associated with urinary tract infection (UTI). The magnitude of the association of diaphragm use with UTI was reduced when urination habits around sexual intercourse were considered.
\end{abstract}

Urinary tract infection Behavior Birth control Determinants Diet Epidemiology Sexual intercourse

\section{INTRODUCTION}

A variety of behavioral factors have been associated with urinary tract infections (UTI) in women. Although several studies have found that sexual intercourse and diaphragm use increase risk of infection among young women [1-5], few have studied the effects of diaphragm type, and frequency and length of diaphragm use $[3,5]$. The role of other factors, such as urination habits, clothing, menstrual protection and diet, is less clear [4-7]. Moreover, the interactions of these other factors with sexual intercourse and diaphragm use have yet to be described.

In the female, the primary route by which bacteria gain entrance into the urinary tract infection is by ascendance [8]. Therefore, pathogenic bacteria must first colonize the periurethral region. In women with recurring

*This work was supported by Grant No. DK 35368 from the National Institute of Diabetes, Digestive and Kidney Diseases. infections, Stamey [9] has shown that the introitus is first colonized with bacteria. Thus, factors that change the vaginal flora, such as diaphragm use [10], might increase risk of infection.

UTIs tend to recur. The frequency of a new UTI is greater among women who have had a previous infection than among women without such history [11]. Therefore, the relative importance of the different risk factors may vary between first and subsequent infection [7]. In addition, women may change their habits in response to first infection, making it more difficult to determine the effects of potential risk factors on recurrence.

This paper presents the results of an incidence-density case-control study designed to test the association of several hypothesized risk factors and UTI. The overall goals of the study are first, to better understand the etiology of UTI; and second, to identify behaviors which a woman with UTI might adopt or modify to minimize the risk of recurring UTI. 


\section{METHODS}

\section{Case selection}

Cases were recruited from students using the University of Michigan Health Service, a large ambulatory facility serving the needs of 35,000 students, their families, and University faculty and staff. Services are provided free of charge to students. The Health Service sees $60 \%$ of the student population at least once annually. In any 1 year, $2 \%$ of the over 100,000 visits are for UTI (this includes the initial and all related visits, including follow-up urine cultures).

All women presenting to the University Health Service between September 1986 and December 1987 with urinary symptoms-painful, urgent, or frequent urination, or blood in the urine (potential cases) - were offered a selfadministered questionnaire by the triage nurse. A total of 1065 women meeting these criteria were asked to participate; $515(48.4 \%)$ returned the study questionnaire. Of the questionnaires returned, 33 were blank, 3 women refused because they had completed a questionnaire on a previous visit, and 11 women did not give consent, leaving 468 complete questionnaires.

\section{Control selection}

We selected two different control groups: Health Service controls, and population controls. Health service controls were sampled from all women presenting to the Health Service during the same time period as the cases. For each potential case, three questionnaires were distributed to women not reporting urinary symptoms. A total of 3191 questionnaires were distributed of which $1717(53.8 \%)$ were returned. 131 questionnaires were returned blank; 8 had illegible signatures. 29 women refused, $(10$ because of prior enrollment), and 65 women did not consent, leaving 1484 ( $46.5 \%$ of those distributed) potential controls.

Population controls were used to assess whether users of the Health Service were indeed representative of all students with respect to the variables under study. Thus, the sample size was much smaller than that of the Health Service control group. Population-based controls were selected from a list of all students enrolled at the University during January 1988. This constitutes virtually the entire population eligible to enroll in the study. To appear on the list, students must not be refused to have their names appear in a student directory. The sample was inadvertently enriched for out-of- state students. A random sample stratified by year in school (freshman, sophomore, junior, senior, graduate), was selected. 40 students were selected from each strata; 170 had complete and accurate addresses. These students were sent a self-administered questionnaire whose content was the same as that distributed at the Health Service. Because these students may have not been seen recently at the Health Service, and our previous work showed self-report of previous UTIs to have excellent agreement with medical records [3], medical records were not reviewed for this group. Up to three questionnaires were sent to each potential participant. 132 participants returned the questionnaire; among these, 16 refused. One woman reported currently having a UTI and was excluded, leaving a total of 115 population-based controls in the study.

\section{Study questionnaire}

Cases and controls completed a self-administered questionnaire which included questions on medical history, stress, clothing, diet, sexual activity and birth control method during the previous 4 weeks. To avoid potential selection biases, the questionnaire was labelled $A$ Survey of Personal Habits and Infections in Women. Therefore, a woman's decision to participate should not have been influenced by her own feelings regarding UTI research.

\section{Medical record review}

Medical records of all potential cases and Health Service control were reviewed. The following information was abstracted from all records in which the record and the corresponding visit date to the questionnaire completion date could be located $(98.6 \%)$ : diagnosis at time of enrollment, history of UTI, birth control method-including size and type of diaphragm, and/or type of oral contraceptive prescribedand for potential cases, results of urinalysis, urine culture and antibiotic sensitivities. With the exception of diagnosis, these data were used primarily to validate the information received in the self-administered questionnaire. Medical records of population controls were not reviewed (see above).

\section{Case and control definitions}

Women were eligible to participate if they were currently enrolled at the University, were not known to be pregnant, and did not report a recent history of hospitalization or catheterization. 
A UTI was defined as the presence of significant bacteriuria and one or more urinary symptoms: painful urination, frequent urination, urination at night, urgent need to urinate or blood in the urine. Analyses were carried out using two different definitions of significant bacteriuria: $\geqslant 100,000$ colonies (col) of bacteria $/ \mathrm{ml}$ urine and $\geqslant 10,000$ col of a single bacteria/ml urine. The second definition of significant bacteriuria was considered for two reasons: first, Stamm et al. [12] suggested that among symptomatic women, $\geqslant 100$ bacteria $/ \mathrm{ml}$ urine was the best diagnostic criterion; and second, the clinical laboratory used did not routinely enumerate bacteria occurring less frequently than $10,000 / \mathrm{ml}$ urine and the study lacked funds to carry out additional laboratory analyses. A total of 3 women (1\%) with urinary symptoms who otherwise met study criteria were excluded because they had less than 10,000 bacteria but greater than zero bacteria/ml urine. Using the more specific criteria of $\geqslant 100,000 \mathrm{col}$ bacteria $/ \mathrm{ml}$ resulted in the exclusion of an additional 24 cases with no prior UTI and 47 cases with one or more prior UTIs. Changing the definition of bacteriuria made virtually no difference in study findings; thus only results using the standard definition $\geqslant 100,000 \mathrm{col}$ bacteria/ml of urine are presented here.

Urinalyses and urine cultures were performed by the University Health Service laboratory using standard techniques. Patients were instructed in obtaining a clean catch midstream specimen. Routine urine culture included inoculation of MacConkeys, Blood and Phenylethanol agar plates with a measured amount of urine. Plates were allowed to incubate at $37^{\circ} \mathrm{C}, \mathrm{CO}_{2}$, overnight. Bacterial colonies with $\geqslant 10,000$ col bacteria $/ \mathrm{ml}$ urine were identified by subculture and biochemical tests. Antibiotic sensitivities were performed only upon physician request.

A first UTI was defined as the first occurrence of a urinary tract infection. A history of prior UTI(s) was defined by report either on the medical record or on the self-administered questionnaire [Did a health care provider ever say you had a kidney, bladder or urine infection (for example, cystitis, or pyelonephritis)?]. Agreement between self-report and the medical record was extremely high $(99.4 \%)$; in the 11 cases where there was a discrepancy, self-report was taken as truth. If no history of UTI was present on medical records, self-report of UTI history was taken as truth.
Controls were defined as women without UTI (as defined above). For population controls, self-report of current UTI was deemed sufficient to exclude the woman from the study. Because a first UTI is a risk factor for repeated UTIs, comparisons were made between women with first UTI and controls without a history of UTI. Women with a second UTI were compared to women with a history of one UTI, and so forth.

\section{Analysis}

The associations between the various risk factors and UTI were studied using multilevel contingency tables, with both stratification and multivariate techniques to control for confounding [13]. All comparisons were made using Health Service Controls and population-based controls separately. Since many of the hypothesized risk factors for vaginal infections are similar to UTI risk factors, Health Service Controls were stratified by clinical diagnosis at time of enrollment to determine whether excluding women and vaginal infections affected study findings. Because women with vaginal infections were not different from other controls, they are included in the analyses presented here.

Analyses were done separately for women with none and one or more prior UTIs. A detailed analysis of the interactions between number of previous UTIs, birth control method and frequency of sexual intercourse controlling for all other risk factors using Miettinen's confounder score [14] convinced us of the importance of separating women by UTI history. Merely including an indicator of prior UTI history in the multivariate analyses distorted the observed results relative to separate analyses.

To calculate odds ratios (ORs) controlling for the confounding effects of age, frequency of sexual intercourse, diaphragm use, report of a local personal physician outside the Health service, and month of enrollment (hereafter, confounding variables), a separate logistic regression model was fit for each important risk factor identified in the stratified analysis. Related variables, such as birth control methods, were fit in a single model. Separate models were fit for the cases compared with population controls and for cases compared with Health Service controls. Reported ORs and their $95 \%$ confidence intervals (CIs) were calculated from the logistic regression model as described by Schlesselman [13]. For example, the OR for tampon use for cases compared to Health Service controls was calculated from a logistic 
regression model with presence of UTI as the dependent variable, and age, frequency of sexual intercourse, diaphragm use, month of enrollment, and indicators for tampon use, tampon and napkin use, and using no menstrual protection (sanitary napkin use was the referent). For women with a history of at least one prior UTI, an indicator of the number of previous UTIs was included in the model. Results comparing cases to population controls are not shown, as the findings were consistent with those found using Health Service controls.

\section{RESULTS}

\section{Description of study population}

A total of 2067 women, 468 potential cases (women with urinary symptoms), 1484 potential Health Service controls, and 115 potential population controls enrolled in the study. Medical records could not be reviewed for 2 potential cases and 29 potential Health Service controls. In addition, a total of 324 women were excluded: 7 were pregnant, 19 had been recently hospitalized, 7 catheterized, 61 had missing data on UTI history, and 252 were not students. Moreover, 147 (31.4\%) of the otherwise eligible cases did not have greater than $100,000 \mathrm{col}$ bacteria/ml urine in a single midstream urine culture; of these 73 had no bacteria present. Pyuria, defined as 10 or more leukocytes $/ \mathrm{mm}^{3}$, was present in $83 \%$ of the cases; $83 \%$ of the positive cultures were coliforms. This left a final sample size of 1641 women: 237 cases, 1296 Health Service controls, and 108 population controls. (Some women were excluded for multiple reasons so the total number excluded do not add to 324.)

Participants averaged 21.9 years of age with a standard deviation of 4.4 years. About twothirds of the cases $(63.2 \%)$, one-third of the Health Service controls (36.4\%), and onequarter of the population-based controls $(25.2 \%)$ reported a history of prior UTI. Participants tended to answer all questions: completion rates per question ranged from $99.0 \%$ for questions regarding sexual activity, to $99.5 \%$ for questions regarding diet. When cases and controls were stratified by UTI history, cases and Health Service controls were similar with respect to year in school, age, marital status, ethnic group, and presence of a private personal physician outside of the Health Service. Population-based controls were younger and less likely to have a private physician than cases. Analyses using population-based controls were consistent with those for Health Service controls; thus, only results using Health Service controls are presented here.

Study participants were similar to women students enrolled in the University with respect to year in school, age, ethnic group and marital

Table 1. Distributions of selected variables by history of previous UTI

\begin{tabular}{|c|c|c|c|c|c|c|c|c|}
\hline \multirow[b]{3}{*}{ Variable } & \multicolumn{4}{|c|}{ No prior UTIs } & \multicolumn{4}{|c|}{ One or more prior UTIs } \\
\hline & \multicolumn{2}{|c|}{$\begin{array}{c}\text { Cases } \\
(n=86)\end{array}$} & \multicolumn{2}{|c|}{$\begin{array}{l}\text { Controls } \\
(n=816)\end{array}$} & \multicolumn{2}{|c|}{$\begin{array}{c}\text { Cases } \\
(n=151)\end{array}$} & \multicolumn{2}{|c|}{$\begin{array}{l}\text { Controls } \\
(n=480)\end{array}$} \\
\hline & $n$ & $\%$ & $n$ & $\%$ & $n$ & $\%$ & $n$ & $\%$ \\
\hline \multicolumn{9}{|l|}{ Frequency of sexual intercourse/week } \\
\hline none & 3 & 3.5 & 433 & 53.1 & 7 & 4.6 & 131 & 27.3 \\
\hline$<1$ & 18 & 20.9 & 117 & 14.3 & 17 & 11.3 & 83 & 17.1 \\
\hline $1-2$ & 30 & 34.9 & 130 & 15.9 & 43 & 28.5 & 116 & 24.2 \\
\hline $3-6$ & 28 & 32.6 & 115 & 14.1 & 70 & 46.4 & 121 & 25.2 \\
\hline$\geqslant 7$ & 7 & 8.1 & 21 & 2.6 & 14 & 9.3 & 30 & 6.3 \\
\hline \multicolumn{9}{|l|}{ Sexually active women only } \\
\hline New partner & 14 & 15.0 & 45 & 12.0 & 25 & 17.5 & 41 & 12.0 \\
\hline \multicolumn{9}{|c|}{ Birth control method (categories not mutually exclusive) } \\
\hline Oral contraceptives & 34 & 42.0 & 215 & 57.5 & 68 & 47.6 & 183 & 53.2 \\
\hline Diaphragm use (rare, some, & & & & & & & & \\
\hline frequently, or always use) & 28 & 34.6 & 31 & 8.3 & 43 & 30.1 & 78 & 22.7 \\
\hline Spermicides & 31 & 40.3 & 61 & 16.5 & 55 & 40.4 & 99 & 29.6 \\
\hline All other methods & 40 & 52.0 & 151 & 40.8 & 50 & 36.8 & 125 & 37.3 \\
\hline \multicolumn{9}{|c|}{ Urinating within $15 \mathrm{~min}$ after sexual intercourse } \\
\hline always & 8 & 9.9 & 60 & 16.0 & 22 & 15.4 & 77 & 22.3 \\
\hline frequently & 18 & 22.2 & 87 & 23.1 & 38 & 26.6 & 108 & 31.3 \\
\hline some & 25 & 30.8 & 112 & 29.8 & 37 & 25.9 & 92 & 26.7 \\
\hline rare & 14 & 17.3 & 87 & 23.1 & 36 & 25.2 & 54 & 15.7 \\
\hline never & 16 & 19.8 & 30 & 8.0 & 10 & 7.0 & 14 & 4.1 \\
\hline
\end{tabular}


status. Some women completed all or part of the questionnaire but did not consent to participate, i.e. they did not consent to a review of their medical records. When compared to participants, these refusers were more likely to be married and non-white than participants, but the differences were not large and did not reach statistical significance at the 0.05 level.

\section{Associations between risk factors and UTI}

Sexual intercourse and birth control method. As expected, women with UTI were more likely to have engaged in sexual intercourse during the previous 4 weeks than controls (Table 1). When controlled for age, frequency of diaphragm use, report of a local personal physician outside the Health Service and month of enrollment, we observed a dose-response relationship between frequency of sexual intercourse and UTI when comparing each group to their respective control groups (Table 2). That is, the odds of exposure among cases compared to the odds of exposure among controls, increased with increasing frequency of sexual intercourse. For both case groups and for all frequencies of exposure, the odds ratio was strong and statistically significant at the 0.05 level-even at the lowest level of exposure (Table 2).

After adjusting for frequency of sexual intercourse and other confounding variables, there was only a weak association between starting a new sexual relationship during the past 4 weeks and UTI (Table 2). There was no apparent association between self-report of multiple sexual partners during the past 4 weeks and UTI [No prior UTIs: $O R=0.63(0.21,1.86)$; one or more prior UTIs: $O R=1.04(0.52$, 2.07)].

We asked about all birth control methods used during the 4 weeks prior to enrollment. Some sexually active women used multiple methods, thus the categories in Table 1 are not mutually exclusive. The most frequently reported method was oral contraceptives (Table 1). To examine the association between birth control method and UTI, analyses were limited to women sexually active during the previous 4 weeks. A logistic model was fit controlling for confounding variables, and including indicators for spermicide and other birth control use during the 4 weeks prior to enrollment (oral contraceptives were the reference). Women with no prior UTIs were eight times as likely as controls to always have used a diaphragm with spermicides compared to oral contraceptives (Table 2). Women with one or more prior UTIs were almost twice as likely to always have used a diaphragm with spermicides than oral contraceptives as a birth control method (Table 2).

Table 2. Associations between UTI and sexual intercourse, birth control method and urination habits around sexual intercourse during the previous 4 weeks, by history of UTI, adjusted for age, diaphragm use, month of enrollment, and personal physician outside the Health Service in a logistic regression model

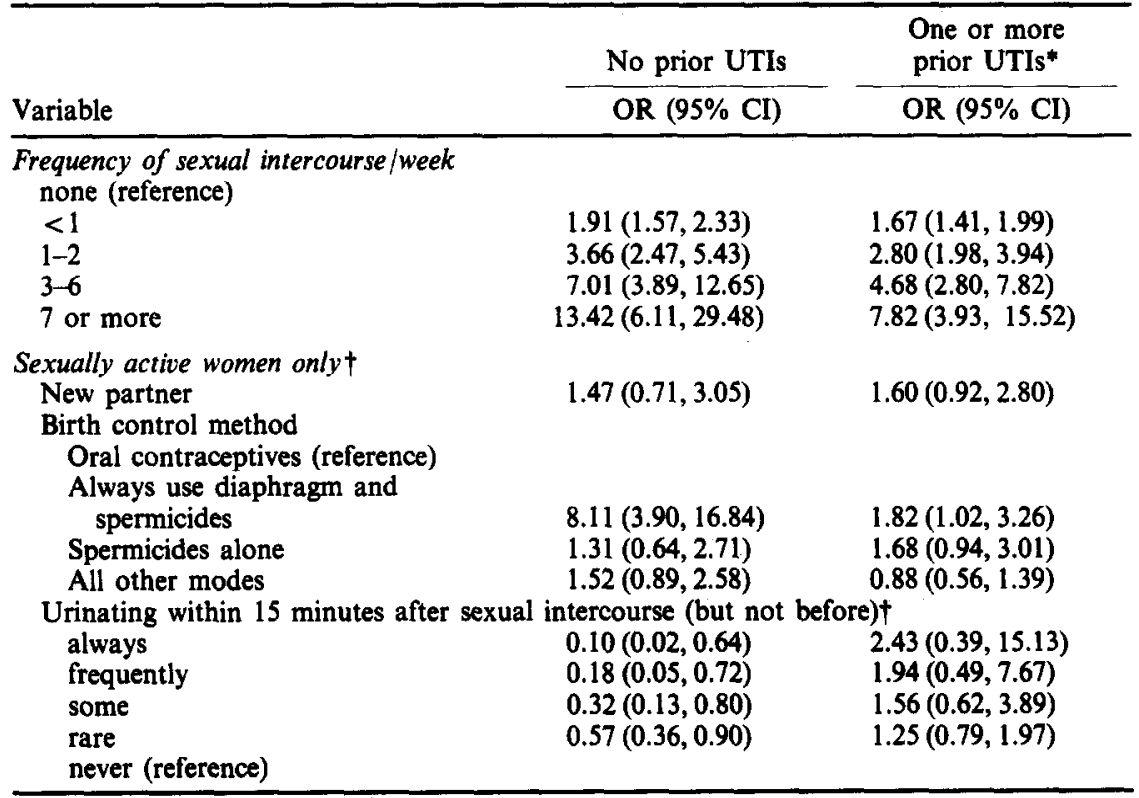

*Also adjusted for number of prior UTIs.

†Also adjusted for frequency of sexual intercourse. 
No one diaphragm type (flat coil, arcing or other) showed an increased association with UTI (data not shown); however the numbers in each group were small. Numbers were also too small to draw any conclusions regarding length of diaphragm wear. Use of spermicides alone had an apparent positive association with UTI independent of diaphragm use. However, after controlling for confounding variables, and other birth control methods, the results did not reach statistical significance at the 0.05 level (Table 2).

Urination habits around sexual intercourse. Urinating before and/or after sexual intercourse is one of the most frequently mentioned preventive measures in the self-help literature [15-18]. The proportion of sexually active women never or rarely urinating before and after sexual intercourse decreased with the number of previous UTIs. Cases were more likely to never or rarely urinate after sexual intercourse than controls, but there was no clear pattern for urinating before sexual intercourse (Table 1).

Whether a women urinates after sexual intercourse is associated with whether she urinates before and with the amount of liquid she typically consumes. On average, women with UTI reported drinking less liquid than controls (daily consumption: $\quad$ cases $=1.56$ liters, controls $=$ 1.64 liters; $t=1.09, \quad d f=1557, \quad p=0.27$ ). Among women sexually active during the past 4 weeks, women with UTI were less likely to urinate after sexual intercourse than controls (Table 1).

We fit a logistic regression model to the data controlling for confounding variables, total liquid consumed, urinating before and after sexual intercourse, and an interactive term between urinating before and urinating after sexual intercourse. For women with no prior UTIs, always urinating after sexual intercourse compared to never doing so was protective $[O R=0.10(0.02,0.64)]$. The association was strong even when the habit was done rarely (Table 2). Always urinating before sexual intercourse compared to never doing so [OR $=0.34$ $(0.05,2.16)]$ and always urinating both before and after sexual intercourse [OR $=0.29(0.04$, 2.24)], also had apparent protective effects, but the confidence intervals are wide and the results are not statistically significant at conventional levels.

The results for women with a history of one or more UTIs conflict with those for women with no prior UTI. This may reflect a bias, in that women with prior UTI may be more likely to perform the behavior in response to symptoms or be more likely to recall doing so. In any case, the confidence intervals are wide and the results are not statistically significant at conventional levels (Table 2). Further, the association with urinating both before and after sexual intercourse $[O R=0.48(0.17,1.40)]$, while not reaching conventional levels of statistical significance, is consistent with the findings among women with no prior UTIs.

Urination habits and diaphragm use. We also examined the joint effects of diaphragm use and urinating before and/or after sexual intercourse on risk of UTI. When adjusted for urination habits in a logistic regression model which included confounding variables and birth control methods, using a diaphragm with spermicide compared to oral contraceptives remained a risk factor. However, when urination habits were considered, urinating both before and after sexual intercourse decreased the association between diaphragm and spermicide use and UTI regardless of UTI history. Although suggestive, these associations did not reach statistical significance at conventional levels. Nonetheless, because of the potential importance of these findings, the results are presented in detail below.

Among women with no prior UTI, using a diaphragm with spermicides and urinating only before sexual intercourse had an OR of 3.41 $(0.48,24.20)$; this is considerably smaller than the observed OR of $9.53(4.46,20.36)$ for diaphragm and spermicide use holding urination habits constant. Using a diaphragm with spermicides and urinating only after sexual intercourse had an OR of $1.17(0.17,8.18)$. Urinating both before and after sexual intercourse and using a diaphragm with spermicides compared to oral contraceptives also showed an apparent decrease in the observed OR for diaphragm and spermicide use [OR $=3.24(0.91$, 11.47)].

A similar analysis among women with one or more prior UTIs who used the diaphragm and spermicides compared to oral contraceptives, also showed urinating before and after sexual intercourse to apparently reduce the OR associated with diaphragm and spermicides [OR $=$ $0.92(0.28,3.00)$ ] compared to $\mathrm{OR}=2.01(1.09$, 3.69) for diaphragm and spermicide use holding urination habits constant. By contrast, in this same group only urinating before or only urinating after sexual intercourse had an increased 
OR [before: $\mathrm{OR}=7.14 \quad(1,18,43.31)$; after: OR $=5.65(2.09,15.27)]$.

Toilet habits, menstrual protection, personal hygiene and clothing. Toilet habits-frequency of urination, waiting before urinating, hesitating to excuse oneself to urinate, wiping back-tofront vs front-to-back after using the toilet, using colored toilet paper, experiencing constipation, and using laxatives or stool softenersshowed no consistent association with UTI after controlling for confounding variables in separate logistic regression models for related sets of variables (for example, menstrual protection was considered in a single model). Tampons and napkins together were the most common form of menstrual protection in all case and control groups, but there was little association between menstrual protection and UTI for any group after controlling for confounding variables.

Feminine hygiene products, tub baths, type of soap, and most clothing types (panty hose, wearing jeans or pants vs skirts or dresses, wearing hose under pants or jeans), and how clothing is worn (tight vs lose) also showed little or no association with UTI, and the relationships were unaffected by control of confounding variables in separate logistic regression models for related variables (for example, all underwear types were considered in a single model). Wearing underwear made of synthetic fiber compared to underwear of cotton only was associated with UTI, even after controlling for confounding variables, but results reached statistical significance only for women with one or more prior UTIs [no prior UTI: OR $=1.29(0.46,3.62)$; one or more prior UTIs: $\mathrm{OR}=2.24(1.03,4.85)]$.

Dietary factors. Examination of the crude associations between UTI and diet during the previous 4 weeks showed most factors-aspirin, acetaminophen, alcohol, soda pop, vegetarian diet, chilli peppers, ginger, garlic, sweets, chocolate, tea, coffee, orange juice, other citrus juice, milk, total liquids consumed and cigarettes-to have little or no association. Controlling for confounding variables, and for tea, coffee and other liquids, total liquid consumed, in separate logistic regression models for each set of related variables, (e.g. spicy foods: chilli peppers, ginger and garlic were included in the same model), changed the crude ORs only marginally.

Taking vitamin $\mathrm{C}$ had an apparent protective effect on the risk of UTI, which remained after adjustment for confounding variables [no prior UTI: $O R=0.59(0.35,0.98)$; one or more prior UTIs: $\mathrm{OR}=0.85(0.58,1.25)]$. The associations with drinking cranberry juice - an age old remedy for UTI-did not reach conventional levels of significance when adjusted for confounding variables and total liquid consumed, in a logistic regression model [no prior UTIs: $O R=1.53$ $(0.61,3.83)$; one or more prior UTIs: $\mathrm{OR}=1.60$ $(0.88,2.89)]$.

Stress. We had four simple measures of stress: not getting enough sleep, having no or only one confidant, an 8 item stress list, and a 10 point rating scale of stress (terrible to wonderful). Only the stress scale showed more than a slight association with UTI. However, women with UTI were most likely to report the lowest levels of stress-even after adjusting for confounding variables [comparing feeling wonderful to feeling terrible, no prior UTIs: $O R=2.71(0.69$, 10.68); one or more prior UTIs: $\mathrm{OR}=2.86$ $(1.06,7.73)]$.

\section{DISCUSSION}

We observed associations between UTI and sexual intercourse, diaphragm use, vitamin $\mathrm{C}$ use, and, for women with no prior UTIs, urinating after sexual intercourse. Other factors, including the direction a women wipes after urination, frequency of urination (other than around sexual intercourse), menstrual protection, diet, clothing, and hygiene were not associated with UTI, consistent with the results of Foxman and Frerichs [7], Remis et al. [4] and Strom et al. [5].

An association between UTI and diaphragm use has been observed in several previous studies $[2-5,19,20]$. However, none of these studies examined the joint effects of diaphragm use and urination habits around sexual intercourse. Strom et al. [5] and others [6, 7] report a protective effect for urinating after sexual intercourse, but do not demonstrate the dose-response relationship found here among women with no prior UTI. Urinating before and/or after sexual intercourse may decrease risk of UTI by flushing out bacteria and removing media for bacterial growth. In fact, when women with no prior UTI were followed for 6 months, the incidence of second infection was lower among women who reported urinating after sexual intercourse [21].

Health education materials and self-help books commonly suggest urinating before and after sexual intercourse as a method of decreasing risk of future UTI [15-18]. Indeed, in our study, women with a history of prior UTI were 
more likely to report urinating after sexual intercourse than those with no such history-to the extent that it is an apparent risk factor in this group. This may reflect a bias, however, in that women with prior UTIs may be more careful about urinating around sexual intercourse when they have early UTI symptoms, or may be more likely to recall doing so.

The diaphragm is an effective, reversible method of birth control with few side effects. Thus, if urination habits around sexual intercourse truly reduce the risk of UTI among diaphragm users, diaphragm users who suffer from multiple UTIs could try modifying their urination habits rather than finding another acceptable birth control method. Although our results did not reach statistical significance at conventional levels, we did observe that urinating after sexual intercourse apparently diminishes the association between UTI and using a diaphragm with spermicides compared to oral contraceptives among women with no prior UTIs, as did urinating both before and after sexual intercourse among women with one or more prior UTIs, even after controlling for frequency of sexual intercourse, age, and other confounding variables.

Unlike previous studies [2-7], we had both a Health Service and a population control group. This allowed us to evaluate the effects of selection bias. Population-based controls were less likely than Health Service controls to be sexually active ( 39 vs $58 \%$ ), have a history of previous UTIs ( 25 vs $38 \%$ ) or to have a private physician within driving distance (5 vs $22 \%$ ). This latter difference may reflect the fact that the population sampled was inadvertently enriched for out-of-state students. However, the results found with each of the control groups were generally similar.

Although not all women with urinary symptoms were asked to participate, and of these, after refusals and exclusions only $29 \%$ were ultimately enrolled as cases in the study, we do not believe the validity of the results were compromised by selection bias. Selection criteria were applied uniformly to cases and controls, resulting in case and control groups similar with respect to demographic variables, year in school and academic major. Moreover, there were no differences between cases and controls with respect to variables not expected to be associated with urinary tract infection, such as consumption of non-herbal teas, sweets, or aspirin.
A review of medical records found no cases to be simultaneously infected with chlamydia or gonorrhea. Nonetheless, it is possible that a few cases had undetected infections, biasing upward the observed association between UTI and sexual intercourse. We would expect any bias to be quite small, however, as the association with new sexual parteners was weak and did not reach statistical significance, and there was no apparent association with having multiple sexual partners and UTI.

Frequent and urgent urination, dysuria and hematuria are symptoms that are difficult to ignore. Nonetheless, it is likely that some controls, especially population-based controls, could have included women with UTI. The resulting misclassification should result in a conservative bias, as controls would be more like cases than if classification were perfect. Misclassification of exposure might also have occurred if cases were more likely to recall exposures than controls. Differential recall should be limited since the questionnaire was administered prior to diagnosis and questions were about recent (within the past 4 weeks) exposures.

\section{CONCLUSIONS}

This study suggests that frequent sexual intercourse, diaphragm use and, among women with no prior UTIs, urinating after sexual intercourse are associated with UTI. The association with diaphragm use may be reduced among women who urinate both before and after sexual intercourse, regardless of UTI history. This finding needs further study. If urinating around sexual intercourse is truly sufficient to reduce the association between diaphragm use and UTI, it is a behaviour that can be easily adopted by women.

Acknowledgements - The authors thank the University of Michigan Health Service for their support of this study. Without the efforts of the triage nurses who distributed the questionnaires, the study would not have taken place. Thanks also to James Koopman and MaryFran Sowers for helpful comments on earlier drafts of this manuscript, and to research assistants Ana Carriazo, Patricia Crawford, Carl Li, D. Rebecca Prevots, Cynthia Rusk and Ellen Ward for their help in coordinating and managing the study.

\section{REFERENCES}

1. Nicolle LE, Harding GKM, Preiksaitis J, Ronald AR. The association of urinary tract infection with sexual intercourse. J Infect Dis 1982; 146: 579-583.

2. Fihn SD, Latham RH, Roberts P, Running K, Stamm WE. Association between diaphragm use and urinary tract infection. JAMA 1985; 254: 240-245. 
3. Foxman B, Frerichs RR. Epidemiology of urinary tract infection: I. Diaphragm use and sexual intercourse. Am J Public Health 1985; 75: 1308-1313.

4. Remis RR, Gurwith MJ, Gurwith D, Hargrett-Bean NT, Layde PM. Risk factors for urinary tract infection. Am J Epidemiol 1987; 126: 685-694.

5. Strom BL, Collins M, West SL, Kreisberg J, Weller S. Sexual activity, contraceptive use, and other risk factors for symptomatic and asymptomatic bacteriuria. Ann Intern Med 1987; 107: 816-823.

6. Adatto K, Doebele KG, Galland L, Granowetter L. Behavioral factors and urinary tract infection. JAMA 1979; 241: 2525-2526.

7. Foxman B, Frerichs RR. Epidemiology of urinary tract infection: II. Diet, clothing and urination habits. Am J Public Health 1985; 75: 1314-1317.

8. Sobel JD. Pathogenesis of urinary tract infections. Host defenses. Infect Dis Clin N Am 1987; 1: 751-771.

9. Stamey TA, Timothy M, Millar M et al. Recurrent urinary infections in adult women: the role of introital bacteria. Calif Med 1971; 115: 1-19.

10. Peddie BA, Bishop V, Bailey RR, McGill H. Relationship between contraceptive method and vaginal flora. Aust NZ J Obstet Gynaecol 1984; 24: 217-218.

11. Kunin CM, Polyak F, Postel E. Periurethral bacterial flora in women: prolonged intermittent colonization with Escherichia coli. JAMA 1980; 243: 134-139.
12. Stamm WE, Counts GW, Running KR, Fihn S, Turck M, Holmes KK. Diagnosis of coliform infection in acutely dysuric women. N Engl J Med 1982; 307: $463-468$.

13. Schlesselman JJ. Case-Control Studies: Design, Conduct, Analysis. New York: Oxford University Press; 1982.

14. Miettinen OS. Theoretical Epidemiology: Principles of Occurrence Research in Medicine. New York: John Wiley: 1985: 242.

15. Smith ED. Women's Health Care: a Guide for Patient Education. New York: Appleton-Century-Crofts; 1981: 37, 47-48.

16. Cooke CW, Dworkin S. The Ms Guide to a Woman's Health. New York: Anchor Books; 1979: 317.

17. McPherson A. Women's Problems in General Practice, 2nd edn. Oxford general practice series 12. Oxford: Oxford University Press; 1988: 347.

18. Kilmartin A. Cystitis: the Complete Self-help Guide. New York: Warner Brooks; 1980.

19. Gillespie L. The diaphragm: an accomplice in recurrent urinary tract infections. Urology 1984; 24: 25-30.

20. Vessey M, Doll R, Peto $R$ et al. A long-term follow-up study of women using different methods of contraception-an interim report. J Biosoc Sei 1976; 8: 373-427.

21. Foxman B. Recurring lower urinary tract infections: occurrence and determinants. Am J Public Health 1990; in press. 